\title{
Profile of patients with hepatic hydatid disease not treated surgically
}

\author{
José Manuel Ramiaㄹ, Roberto de-la-Plaza ${ }^{1}$, Miguel Casares $^{2}$, Carmen Ramiro $^{1}$, Pilar Veguillas ${ }^{1}$, \\ José Quiñones $^{1}$ and Jorge García-Parreño ${ }^{1}$ \\ ${ }^{1}$ Unit of Hepato-Bilio-Pancreatic Surgery. Department of General and Digestive Surgery. ${ }^{2}$ Department of Radiology. \\ Hospital Universitario de Guadalajara. Guadalajara, Spain
}

\begin{abstract}
Background: hepatic hydatid disease (HHD) is still an important health problem in certain areas of Spain where it is endemic. The treatment of HHD is usually surgical but certain patients are found to be ineligible after assessment for surgery (asymptomatic disease, comorbidity, patient refusal, or other).

Material and methods: description of patients assessed in the Department of Surgery for hepatic hydatid disease.

Results: in a group of 70 patients with $\mathrm{HHD}, 27$ patients were not treated surgically (mean age: 72.7 years [range: 47-97], 14 women [51.8\%]). The number of cysts presented by these patients was 33, with 1.22 cyst/patient (range: 1-4). The cyst size was 5.5 $\mathrm{cm}$ (range: $2.1-12.5 \mathrm{~cm}$ ). The cysts, according to the WHO classification, were CE1: 3 patients, CE3B: 5 patients, CE4: 10 patients and CE5: 9 patients. The form of presentation was: symptomatic in 9 patients, although only 6 were attributable to HHD (22\%) and asymptomatic in 18 patients. In these cases, imaging was performed for study of tumor extension in 6 patients and diverse medical reasons in 12. Only two therapeutic interventions were performed: endoscopic retrograde cholangiopancreatography (ERCP) with insertion of a bile duct stent, and puncture-aspiration-injection-re-aspiration (PAIR), both in patients who did not wish to undergo surgery.

Ten patients had surgical indications: CE1 (3 patients), CE3B ( 5 patients), CE4 (1 patient), and CE5 (1 patient). The reasons why the patients did not undergo surgical treatment were: refusal ( 9 patients) and advanced neoplasm (1 patient). Surgery was judged necessary in 5 patients. In the mean follow-up period of 17 months (range: 1-37), no surgery was performed.

Conclusions: there were various causes for not performing surgical intervention of HHD after medical evaluation: asymptomatic patients, older patients, patients with multiple pathologies and oncologic patients. Usually, they were patients who voluntarily chose not to undergo surgery.
\end{abstract}

Key words: Hydatid disease. Review. Surgery.

Received: 17-01-11.

Accepted: 08-04-11.

Correspondence: José Manuel Ramia. Unit of Hepato-Bilio-Pancreatic Surgery. Department of General and Digestive Surgery. Hospital Universitario de Guadalajara. C/ Donante de Sangre, s/n. 19002 Guadalajara. Spain. e-mail: jose_ramia@hotmail.com
Ramia JM, de-la-Plaza $R$, Casares M, Ramiro C, Veguillas $P$, Quiñones J, García-Parreño J. Profile of patients with hepatic hydatid disease not treated surgically. Rev Esp Enferm Dig 2011; 103: 448-452.

\section{INTRODUCTION}

Hepatic hydatid disease (HHD) is endemic in certain areas of the planet and is caused by parasites of the genus Echinococcus (1-5). Despite the large number of patients with HHD, the level of evidence on which therapeutic decisions are based is low $(2,4,6)$. While it can be claimed that the mainstay of HHD treatment is surgery, especially when complications exist, other therapeutic options exist, such as pharmacologic treatment with albendazole or cyst puncture with the PAIR technique, an acronym for puncture, aspiration, instillation and re-aspiration, which has become popular for the management of uncomplicated cysts $(1,4)$.

There are no internationally admitted guidelines for the treatment of asymptomatic patients and patients not candidates for surgery (3). Little information exists in the literature about how many patients diagnosed of HHD are finally not treated surgically and what are the reasons for making this decision. Among the possible causes are: surgeon-dependent reasons, the patient's personal decision, comorbidities, cyst characteristics, and other reasons.

\section{OBJECTIVE}

The primary aim of this study was a cross-sectional analysis of the patients diagnosed of hepatic hydatid disease in the Hepato-Bilio-Pancreatic Unit of our center who did not undergo surgery describing the reasons for this decision and the events related to the follow-up of these patients. 


\section{MATERIAL AND METHODS}

The health area of Hospital Universitario de Guadalajara had an estimated population as of 1 October 2010 of 245,026 inhabitants (125,548 men and 119,478 women), according to the Spanish National Institute of Statistics. We made a prospective study of a population that included all the patients with hepatic hydatid disease studied in the Hepato-Bilio-Pancreatic Surgery Unit (HBP Unit) from May 2007 to December 2010 in the health area mentioned. The diagnosis of HHD was made in all the patients by means of sonography, abdominal CT and indirect hemagglutination serology. The variables studied were: age, sex distribution, number of cysts, size of the largest cyst, cyst location, type of cyst according to the WHO classification: CE1, CE2 and CE3b (active), CE3a (transitional), and CE4 and CE5 (inactive) $(7,8)$, clinical form of presentation (asymptomatic, jaundice, abdominal pain or other), casual or targeted diagnosis, a new case or recurrence of a previously treated case, ASA grade, the surgeon's therapeutic decision, the patient's final decision and, if both decisions coincided, the type of treatment used. The statistical analysis was made with SPSS 16.0 for Mac.

\section{RESULTS}

Seventy patients with HHD were treated in our unit. Of these 70 patients, 43 were treated surgically, leaving a study group of 27 patients who were not operated on. The patients not treated surgically had a mean age of 72.7 years and a median age of 75 years (range: 47-97). Fourteen were women $(51.8 \%)$. The study group had 33 cysts, with a mean of 1.22 cysts per patient (range: $1-4$, excluding 2 patients with disseminated hydatid disease and, therefore with uncountable cysts), and a median of 1 cyst/patient. The

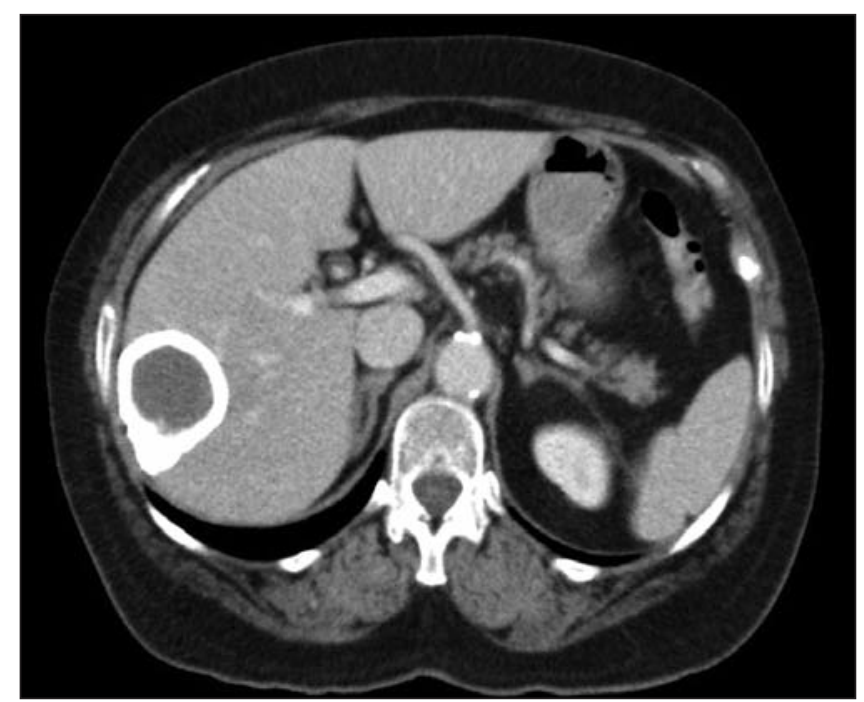

Fig. 1. Asymptomatic CE5 liver hydatid cyst. mean cyst size was $5.5 \mathrm{~cm}$ (range: $2.1-12.5 \mathrm{~cm}$ ) with a median size of $7.5 \mathrm{~cm}$. Based on the WHO classification (7,8): 16 cysts were small $(<5 \mathrm{~cm}), 14$ medium $(5$ to $10 \mathrm{~cm})$ and 3 large $(>10 \mathrm{~cm})(7,8)$. The cyst location was the right liver in 14 patients (48\%), left liver in 5 patients, bilobar in 6 patients, and intra-abdominal dissemination in 2 cases. The cysts were CE1: 3 patients, CE3B: 5 patients, CE4: 10 patients and CE5: 9 patients $(7,8)$ (Fig. 1). CE4 and CE5 cysts were responsible for $70 \%$ of all the cysts in the series. Four patients had recurrences of previously treated HHD.

The clinical form of presentation was: symptomatic in 9 patients (33\%) and asymptomatic in 18 patients. Obstructive jaundice was observed in 4 cases, in 2 patients caused by cysto-biliary communication (Fig. 2), one due to recurrence on the hilar plate of HHD treated surgically in 1996, and 2 for concomitant pancreatic neoplasm with HHD. Another 5 patients had mild abdominal pain, in 4 cases probably caused by HHD, one of whom had disseminated hydatid disease with pain due to obstructive uropathy. The fifth patient had acute pancreatitis due to lithiasis. In the 18 asymptomatic or slightly symptomatic patients, imaging studies were made for: to study the extension of an abdominal neoplasm in 6 patients (4 located in the colon/rectum and 2 in the ovary), all stage IV, for a variety of medical reasons in 11 patients and for follow-up ordered by primary care in an asymptomatic patient with known HHD. Therefore, of the 9 symptomatic patients only 6 ( 2 with jaundice and 4 with abdominal pain) (22.2\%) were attributable to HHD. Forty percent of the patients were diagnosed of HHD casually.

Only two therapeutic interventions were carried out, ERCP with insertion of a bile duct stent in a 77-year-old male patient with hilar recurrence and jaundice who preferred this treatment to surgery, and PAIR in a patient with disseminated hydatid disease and abdominal pain that was finally found to be due to obstructive uropathy cause by a

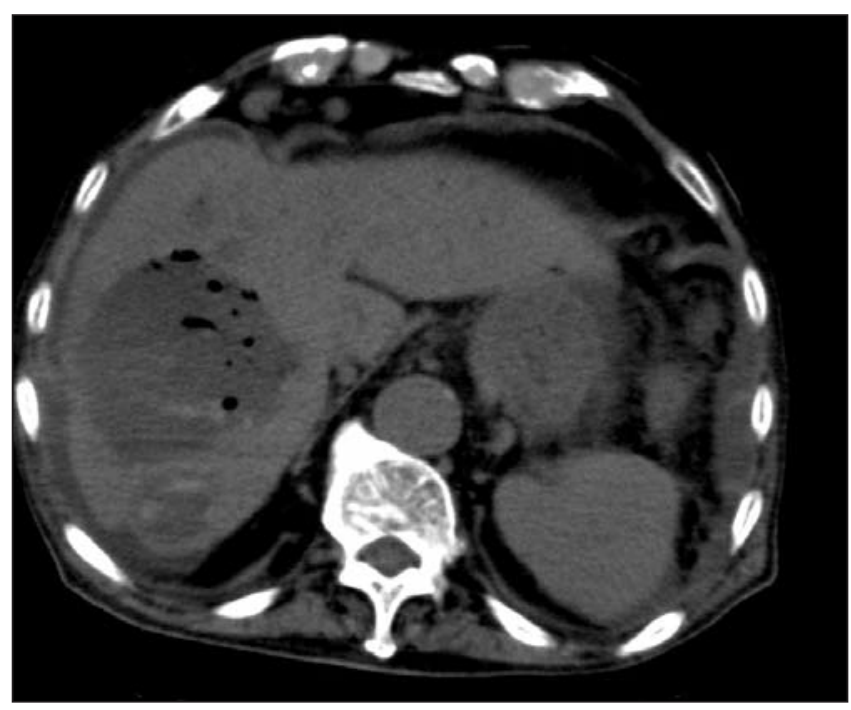

Fig. 2. Cysto-biliary communication in a 97 year-old patient. 
cyst. In both cases, the patients did not want to undergo surgery.

If the surgical indication is based on the radiologic cyst characteristics and WHO recommendations, all the patients with CE1 (3 patients) and CE3B cysts (5 patients), which were active, and with CE4 (1 patient) and CE5 cysts (1 patient), as complicated cysts, then a total of 10 patients required surgery. The reasons why surgery was not performed, categorized by cyst type, were:

- CE1: in these 3 patients, surgery was not performed because the patient refused. These patients included two patients with disseminated hydatid disease (47 and 81 years old), one of whom had undergone surgery six times and preferred PAIR for the symptomatic cyst, and the other an 81-year-old patient with an asymptomatic cyst. The third patient was 74 years old who decided voluntarily not to undergo surgery.

- CE3B: 5 patients, 4 of whom chose voluntarily not to undergo surgery, including 3 patients aged 87,79 and 72 years with an ASA IV surgical risk, and a 54-yearold, ASA I patient with schizophrenia. The fifth patient was 51 years old and had advanced ovarian cancer, so oncologic treatment was the priority.

- CE4: this 97-year-old man with a cysto-biliary communication refused surgery and ERCP despite jaundice.

- CE5: this patient, already discussed, had recurrence in the hilar plate and chose ERCP with stent deployment.

Of these patients, surgery offered a good risk-to-benefit ratio in 4 patients: a 47-year-old patient with symptomatic disseminated hydatid disease, a 74-year-old patient with a CE1 cyst, a 54-year-old patient with a CE3B cyst and psychiatric pathology, and a patient with a CE5 cyst due to recurrent disease who had jaundice. Our 97-year-old patient with jaundice could have been managed with ERCP. A combination of age, comorbidities and the proposed intervention made the surgical indication questionable in the other 5 patients. Therefore, $50 \%$ of the patients chose not to follow our therapeutic recommendations. Our professional opinion and the patient's refusal were recorded in the medical record, but only the 97-year-old patient who requested voluntary discharge signed a denial of consent form.

The 9 patients with CE4 cysts and the 8 patients with CE5 cysts who did not undergo surgery were asymptomatic; 9 of them were patients of advanced age ( $>75$ years), 2 were ASA IV, and 6 had advanced neoplasms.

In the mean follow-up of 17 months conducted (range: 1-37 months), no therapeutic intervention was performed except for periodic replacement of the biliary stent in the patient with hilar disease recurrence.

\section{DISCUSSION}

Epidemiological data have demonstrated that the number of cases of HHD treated surgically differs considerably from the true incidence of HHD in a specific area because a high percentage of patients with HHD are asymptomatic and unaware of their disease (3). There have been practically no studies of patients diagnosed of HHD and assessed for surgery who finally decided to forgo surgery and the reasons for this decision.

The generalized use of imaging tests for the diagnosis of a variety of abdominal diseases has increased the casual diagnosis of asymptomatic cases of HHD, especially in areas of endemic hydatid disease (3). Moreno et al. reported $15.2 \%$ of casual cases (5), which rose to $40 \%$ in our series, perhaps because we work in an area of endemic hydatid disease.

The reason why a high percentage of patients with HHD are asymptomatic for a long period of time and are diagnosed at an advanced age is that the hepatic parenchyma surrounding the hydatid cyst generally offers resistance to cyst growth. Consequently, the cyst grows slowly or not at all for years. Moreover, the liver is very tolerant of the cystic lesion (3).

Therefore, in daily clinical practice we evaluate patients with no symptoms or few symptoms who satisfy one or more of these characteristics: casual diagnosis, generally advanced age, comorbidities or recently diagnosed tumors. The first thing we have to do is to determine whether the symptoms in patients with mild abdominal symptomatology are due to HHD or other causes in order to distinguish between asymptomatic or non-complicated symptomatic cysts (3).

The correct therapeutic decision in these patients is not simple; it is traditionally based on certain characteristics of the cyst and the patient. In an attempt to develop a protocol for action, several classifications have been made and used as an indirect way of evaluating cyst viability and the risk of developing complications before deciding on the treatment for each type of cyst (3).

At present, two HHD classifications are used $(3,8)$. The older classification was developed by Gharbi et al. (10) in 1981 and is based on sonographic findings. In 1995, the WHO published a second classification designed to have prognostic implications and help in clinical decision-making $(7,8)$. In this classification, all the CE1 to CE3 cysts and CE4 and CE5 complicated cysts must be treated surgically, whereas the recommended policy in the non-complicated, theoretically inactive CE4 and CE5 cysts is to "wait and see" and monitor the cysts periodically with sonography $(3,8,9)$. This is the policy followed in our unit.

Calcifications were traditionally thought to be present only in CE5 cysts, i.e. inactive cysts. However, the presence of calcifications has been reported in all types of cysts $(3,8,11)$. This confirms that the classic idea that all calcified cysts are inactive is untrue. Certain authors defend the idea that the solidification of the cyst content and disappearance of vesicles and septa predicts inactivity better than calcification (12). Although wall calcification provides information about the involution of the cyst, only totally calcified cysts can be considered inactive $(3,13)$. 
Ignorance of the natural history of a hydatid cyst and the absence of randomized clinical trials on the long-term follow-up of patients with asymptomatic HHD imply that there is no scientifically valid answer to the question of whether we should treat asymptomatic patients and patients with non-complicated cysts (3). The absence of complications in untreated patients followed up for prolonged periods and the occurrence of spontaneous regressions favor the decision to avoid surgery. In contrast, the difficulty of predicting long-term prognosis and the possibility of developing complications due to cyst growth, some of which may be life-threatening, suggests that asymptomatic young patients should be treated (3). At present, patients with asymptomatic cysts can be managed in different ways: no treatment (watch-and-see policy) or treatment with albendazole, PAIR or surgery $(3-5,8)$. Each option is briefly summarized below.

Treatment with albendazole cannot be considered an ideal option as the only therapy for HHD (4). In addition, albendazole can cause hepatotoxicity and is contraindicated in chronic patients with liver disease (3). The most accepted indications for albendazole are: inoperable cysts, disseminated hydatid disease, small deep cysts, after conservative surgery, for recurrences and in preparation for surgery or PAIR (3). In the study by Moreno et al., 30\% of patients were not treated surgically. Half of the patients in this study were treated with albendazole and the others remained untreated, but no information is provided on the results obtained (5). The combination of PAIR and albendazole in patients with Gharbi 1-3 and CE1 and CE3a cysts seems to achieve safe and effective results. However, a meta-analysis comparing PAIR and surgery excluded the most numerous series (14) due to serious methodological defects and only a short follow-up of patients with PAIR, and thus is based on only two articles $(8,14)$. CE2 and CE3b cysts tend to recur when treated by PAIR (8). Despite the theoretical advantages of PAIR, the technique has not become popular in industrialized countries (5).

Surgery continues to be considered the mainstay of HHD treatment $(3,15,16)$. According to the $\mathrm{WHO}$, the indications for surgery are large cysts with multiple daughter vesicles, superficial single cysts at risk of rupture, infected cysts, and cysts communicating with the bile tract or other organs $(3,9)$. Contraindications for surgery are: pregnant women, asymptomatic patients, severe comorbidities, very small cysts, and multiple or difficult-to-access cysts $(2,3)$. Nonetheless, not all groups agree with these indications, including our group, because these guidelines are adapted to the health care standards of underdeveloped countries where certain treatment options are too expensive. In our opinion, asymptomatic patients with active cysts should undergo surgery and the presence of multiple cysts or difficult access is not a contraindication per se.

At present, no international consensus exists regarding the surgical technique of choice in HHD (conservative versus radical surgery) $(15,16)$. Defenders of conservative techniques praise their simplicity and acceptable results in a large percentage of patients. The defenders of so-called radical surgery (total cysto-pericystectomy or hepatectomy) value its lower morbidity, especially biliary, lower percentage of recurrences, shorter mean stay and the fact that it resolves the failures of conservative surgery $(8,15,16)$. Published series on the surgical treatment of HHD consist of patients treated fundamentally by conservative surgery. At present, HBP surgery units can provide radical surgery figures of almost $75 \%$ with a very low morbidity and mortality, which is why earlier published data do not reflect current reality.

Studying our cases in detail, we observed that according to the criterion of not performing surgery on non-complicated type CE4 and CE5 cysts, only 10 patients had a surgical indication. When we grouped the causes of non-intervention, the decision of the patient in 9 cases to refuse surgery for fear of a negative result due to advanced stage, comorbidities and/or various previous laparotomies implied a high surgical risk. In only one oncologic patient did the members of the unit give priority to treating the tumor instead of the HHD. In 4 cases in which we believed that the operation was advisable but the patient chose to forgo surgery, the patients have not required the surgical treatment offered to date. In a fifth case, a 97-year-old man with jaundice due to frank cysto-biliary communication, only ERCP was offered with the aim of emptying the hydatid content that obstructed the bile tract, as described above (17). However, the patient refused any treatment. The 17 patients with CE4 and CE5 cysts were informed of the diagnosis of HHD, of the likely inactivity of the lesion and, considering their age, of the comorbidities or concomitant diagnosis of neoplasm. None asked for treatment. What is more, the majority confirmed that if they had been offered surgery they would not have accepted it. None of these patients required surgery, as could be expected from patients with inactive cysts.

In conclusion, the treatment of patients with asymptomatic HHD is a topic that is still debated. In young patients with active cysts (CE1 to CE3) and no comorbidities, surgery, radical if feasible, seems to be the best option and there is no consensus regarding what to do with inactive cysts. In asymptomatic patients of advanced age, who frequently have serious comorbidities, or in patients who are diagnosed of HHD in the course of an extension study of a tumor, it seems that even in the case of active cysts there is no evidence of a therapeutic benefit and the patients themselves do not want to undergo surgery.

\section{REFERENCES}

1. WHO/OIE Manual on Echinococcosis in Humans and Animals: a Public Health Problem of Global Concern. Available at: http://whqlibdoc.who.int/publications/2001/929044522X.pdf

2. Brunetti E, Kern P, Vuitton DA and Writing Panel for the WHO-IWGE Expert consensus for the diagnosis and treatment of cystic and alveolar echinococcosis in humans. Acta Tropica 2010;114:1-16.

3. Frider B, Larrieu E. Treatment of liver hidatidosis: how to treat an asymptomatic carrier? World J Gastroenterol 2010;16:4123-9. 
4. Dziri C, Haouet K, Fingerhut A. Treatment of hydatid cyst of the liver: where is the evidence? World J Surg 2004;28:731-6.

5. Moreno J, Tellez CJ, Pardo FJ. Casos de hidatidosis en el Departamento de Salud de la Comunidad Valenciana. Rev Esp Quimioter 2009;22:6267.

6. Dziri C, Haouet K, Fingerhut A, Zaouche A. Management of cystic echinococcosis complications and disemination. World J Surg 2009;33:1266-1273.

7. Junghanss T, Menezes da Silva A, Horton J, Chiodini PL, Brunetti E. Clinical management of cystic Echinococcosis: State of the art, problems and perspectives. Am J Trop Med Hyg 2008;79:301-11.

8. Brunetti E, Junghanss T. Update on cystic hydatid disease. Curr Opin Infect Dis 2009;22:497-502.

9. WHO. Informal Working Group on echinococcosis. Guidelines for treatment of cystic and alveolar echinococcosis in humans. Bulletin WHO 1996;74:231-42.

10. Gharbi HA, Hassine W, Brauner MW, Dupuch K. Ultrasound examination of the hydatic liver. Radiology 1981;139:459-63.
11. Stoupis C, Taylor H, Paley MR, Buetow PC, Marre S, Baer HU et al The rocky liver: radiologic pathologic correlation of calcified hepatic masses. Radiographics 1998;18:675-85.

12. Frider B, Larrieu E, Odriozola M. Long-term outcome of asymptomatic liver hidatidosis. J Hepatol 1999;30:228-31.

13. Rogan MT, Hai WY, Richardson R, Zeyhle E, Craig PS. Hydatid cysts: does every picture tells a story? Trends Parasitol 2006;22:431-8.

14. Smego RA, Bhatti S, Khaliq AA. Percutaneous aspiration-injectionreaspiration drainage: a meta-analysis. Clin Infect Dis 2003;37:107383.

15. Priego P, Nuño J, Lopez Hervás P, Lopez Buenadicha A, Peromingo R, Die J, et al. Hepatic hydatidosis. Radical vs. conservative surgery: 22 years of experience. Rev Esp Enferm Dig 2008;100:82-5.

16. Ramia JM, Figueras Felip J. Hidatidosis hepática: que técnica debemos realizar? Cir Esp 2010;88:1-2

17. Guerrero MT, Prado F, Macias MC, Muñoz A, Ridruejo E, PerezMiranda M. Un anciano con ictericia colestásica por hidatidosis biliar. Rev Esp Geriatr Gerontol 2008;43:52-4. 